International Journal of Pure and Applied Mathematics

Volume 97 No. 4 2014, 419-429

ISSN: 1311-8080 (printed version); ISSN: 1314-3395 (on-line version)

url: http://www.ijpam.eu

doi: http://dx.doi.org/10.12732/ijpam.v97i4.4

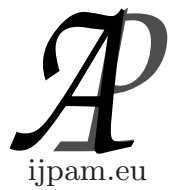

\title{
SOME MULTIPLICATIVE PRODUCT OF $n$-DIMENSIONAL DISTRIBUTIONS. PART II
}

\author{
Rubén Alejandro Cerutti \\ Facultad de Ciencias Exactas \\ Universidad Nacional del Nordeste \\ Avda. Libertad 5540, (3400) Corrientes, ARGENTINA
}

\begin{abstract}
The method of approximants delta sequences, the convolution and the change of variables is used to obtain multiplicative products of $n$ dimensional distributions.
\end{abstract}

AMS Subject Classification: $46 \mathrm{~F} 10$

Key Words: distributional products, delta sequences, change of variables in distributions

\section{Introduction}

This the second article in a series of two devoted to product of distributions obtained through the use of delta sequences and thus a change of variables. This way of work allows that products of one-dimensional distributions are generalized.

In order to obtain product of n-dimensional distributions we will use an uni-dimensional delta sequences in $\mathbb{R}$ and then by changing variables will be obtained the desired generalizations. The method of the delta sequences that have been actively used by B. Fisher, essentially involves a sequence of functions starting from a fixed function $\mathrm{g}$ belonging to the space D of infinitely differentiable functions with compact support as follows. If $S$ and $T$ are distributions, their multiplicative product is defined by the formula

Received: December 24, 2013

(c) 2014 Academic Publications, Ltd. url: www.acadpubl.eu 


$$
S . T=\lim _{n \rightarrow \infty}\left\{S * g_{n}(x)\right\}\left\{T * g_{n}(x)\right\}
$$

if the limit exists for every mollifier $g_{n}(x)$. The symbol $*$ denotes, as usual, convolution. The functions so defined form a sequence of functions in $\mathcal{D}$ which converge to the Dirac delta. By a mollifier we mean a sequence $g_{n}(x)=n g(n x)$, where the function has the properties

1. $g(x) \geq 0$

2. $g(x) \in C_{0}^{\infty}$

3. $\int_{-\infty}^{\infty} g(x) d x=1$

4. $g(x)=g(-x)$

5. $\sup g(x)=[-1,1]$

6. $g(x)$ is increasing for $-1 \leq x \leq 0$ and decreasing for $0 \leq x \leq 1$.

In this definition we follow Gonzalez Dominguez (cf. [1]) who entered a slight variant of the definition due to Mikusinski. This method and other analogous also shown in the review article by $\mathrm{Li}$ (cf. [3]). In order to give a sense to all products evaluated in the paragraphs II, or to regularize them, we deal with multidimensional generalizations obtained by means of the following change of variables. Let $\phi_{s}$ be a distribution of one variable $s$ and let $u(x) \in C^{\infty}\left(\mathbb{R}^{n}\right)$ be such that the $n$-1-dimensional manifold $u\left(x_{1}, x_{2}, \ldots, x_{n}\right)=0$ has no critical point; $\phi_{n}(x)$ denotes the distribution defined on $\mathbb{R}^{n}$ by the formula (called the Leray formula)

$$
\int_{\mathbb{R}^{\mathrm{n}}} f(x) \phi_{u}(x) d x_{1} \ldots d x_{n}=\int_{-\infty}^{\infty} \phi_{s} d s \int_{u(x)=s} f(x) \omega_{u}(x, d x)
$$

here $\omega_{u}$ is an $n-1$-dimensional form on $u$ defined as follows

$$
d u \wedge d \omega=d x_{1} \wedge d x_{2} \wedge \ldots \wedge d x_{n}
$$

the manifold $u(x)=s$ has the orientation such that $\omega_{u}(x, d x)>0$.

By this way we extend some uni-dimensional distributional multiplicative products to certain kinds of n-dimensional distributions called causal and anticausal distributions. 
Definition 1. Let $x=\left(x_{1}, x_{2}, \ldots, x_{n}\right)$ be a point of the n-dimensional euclidean space $\mathbb{R}^{n}$. Let $P(x)$ be a non degenerate quadratic form in $n$ variables of the form

$$
P=P(x)=x_{1}^{2}+\ldots+x_{p}^{2}-x_{p+1}^{2}-\ldots-x_{p+q}^{2}
$$

where $n=p+q$.

The distributions $(P \pm i 0)^{\lambda}$ are defined by

$$
(P \pm i 0)^{\lambda}=\lim _{\epsilon \rightarrow 0}\left(P \pm i \epsilon|x|^{2}\right)^{\lambda}
$$

where $\epsilon>0,|x|^{2}=x_{1}^{2}+\ldots+x_{n}^{2} ; \lambda \in \mathbb{C}$.

The distributions $\left(m^{2}+P \pm i 0\right)^{\lambda}$ are defined in an analogue manner as the distributions $(P \pm i 0)^{\lambda}$. Let us put $(\operatorname{cf}[4], \mathrm{p} 289)$

$$
\left(m^{2}+P \pm i 0\right)^{\lambda}=\lim _{\epsilon \rightarrow 0}\left(m^{2}+P \pm i \epsilon|x|^{2}\right)^{\lambda},
$$

where $\epsilon$ is an arbitrary positive number, and $m$ is a positive real number.

It is useful to state an equivalent definition of the distributions $\left(m^{2}+P \pm\right.$ $i 0)^{\lambda}$. In this definition appear the distributions

$$
\begin{aligned}
& \left(m^{2}+P\right)_{+}^{\lambda}= \begin{cases}\left(m^{2}+P\right)^{\lambda}, & \text { if }\left(m^{2}+P\right) \geq 0 ; \\
0, & \text { if }\left(m^{2}+P\right)<0 .\end{cases} \\
& \left(m^{2}+P\right)_{-}^{\lambda}= \begin{cases}0, & \text { if }\left(m^{2}+P\right)>0 ; \\
\left(m^{2}+P\right)^{\lambda}, & \text { if }\left(m^{2}+P\right) \leq 0 .\end{cases}
\end{aligned}
$$

We can prove, without difficulty that the following formula is valid ([5], p. $566)$

$$
\left(m^{2}+P \pm i 0\right)^{\lambda}=\left(m^{2}+P\right)_{+}^{\lambda}+e^{ \pm i \pi \lambda}\left(m^{2}+P\right)_{-}^{\lambda}
$$

From this formula we conclude immediately that

$$
\left(m^{2}+P \pm i 0\right)^{\lambda}=\left(m^{2}+P-i 0\right)^{\lambda}=\left(m^{2}+P\right)^{\lambda},
$$

when $\lambda=k=$ positive integer.

The distribution $\left(m^{2}+P \pm i 0\right)^{\lambda}$ are entire distributional function of $\lambda$. This is the principal difference between the distributions, formally analogue $(P \pm i 0)^{\lambda}$ which have poles at the point $\lambda=-\frac{n}{2}-k, k=0,1, \ldots$ It can be proved ( $\operatorname{cf}[5]$ p. 573 , formula (2.14) and p. 575 formula (3.5)) that

$$
\left(m^{2}+P \pm i 0\right)^{-k}=P f\left(m^{2}+P\right)^{-k} \mp i \pi \frac{(-1)^{k-1}}{(k-1) !} \delta^{(k-1)}\left(m^{2}+P\right),
$$




$$
k=1,2, \ldots
$$

The following formula is true for every $\lambda, \mu \in \mathbb{C}$, and $m^{2} \neq 0$,

$$
\left(m^{2}+P \pm i 0\right)^{\lambda}\left(m^{2}+P \pm i 0\right)^{\mu}=\left(m^{2}+P \pm i 0\right)^{\lambda+\mu}
$$

(cf. [6], p. 23, formula (1.3.16)), and

$$
\left[\operatorname{sgn}\left(m^{2}+P\right)\right]\left|m^{2}+P\right|^{\lambda}=\left(m^{2}+P\right)_{+}^{\lambda}-\left(m^{2}+P\right)_{-}^{\lambda},
$$

and

$$
\left|m^{2}+P\right|^{\lambda}=\left(m^{2}+P\right)_{+}^{\lambda}-\left(m^{2}+P\right)_{-}^{\lambda},
$$

and

$$
\begin{array}{r}
\left\{\left(m^{2}+P \pm i 0\right)^{\lambda} \ln ^{r}\left(m^{2}+P+i 0\right)\right\} \cdot\left\{\left(m^{2}+P \pm i 0\right)^{\mu} \ln ^{s}\left(m^{2}+P+i 0\right)\right\} \\
=\left(m^{2}+P \pm i 0\right)^{\lambda+\mu} \ln ^{r+s}\left(m^{2}+P+i 0\right)
\end{array}
$$

for all $\lambda, \mu, \lambda+\mu \in \mathbb{C}$, where

$$
\left(m^{2}+P \pm i 0\right)^{\lambda} \ln ^{r}\left(m^{2}+P+i 0\right)=\frac{\partial^{r}}{\partial \lambda^{r}}\left(m^{2}+P+i 0\right)^{\lambda},
$$

where

$$
\begin{aligned}
\ln \left(m^{2}+P+i 0\right)=\lim _{\epsilon \rightarrow 0}\left(m^{2}+P+i \epsilon|x|^{2}\right) & \\
& =\ln \left|m^{2}+P\right|+i \pi H\left(-\left(m^{2}+P\right)\right)
\end{aligned}
$$

and

$$
H\left(m^{2}+P\right)= \begin{cases}1, & \text { for } m^{2}+P>0 \\ 0, & \text { for } m^{2}+P<0 .\end{cases}
$$

In particular we have, taking into account (1.7) and (1.14) that

$$
\begin{aligned}
\left(m^{2}+P+i 0\right)^{r} \ln \left(m^{2}\right. & +P+i 0) \\
& =\left(m^{2}+P\right)^{r} \ln \left|m^{2}+P\right|+(-1)^{r} i \pi\left(m^{2}+P\right)_{-}^{r} .
\end{aligned}
$$

and

$$
\begin{aligned}
\left(m^{2}+P+\right. & i 0)^{r} \ln \left(m^{2}+P+i 0\right)=\left(m^{2}+P\right)^{r} \ln \left|m^{2}+P\right| \\
& +2(-1)^{r} i \pi\left(m^{2}+P\right)_{-}^{r} \ln \left(m^{2}+P\right)_{-}-(-1)^{r} \pi^{2}\left(m^{2}+P\right)_{-}^{r} .
\end{aligned}
$$


From (1.17) we have

$$
\left(m^{2}+P+i 0\right)^{-r+\frac{1}{2}}=\left(m^{2}+P\right)_{+}^{-r+\frac{1}{2}}+(-1)^{r} i\left(m^{2}+P\right)_{-}^{-r+\frac{1}{2}} .
$$

Then, we obtain

$$
\begin{aligned}
\left(m^{2}+P+\right. & i 0)^{-r+\frac{1}{2}} \ln \left(m^{2}+P+\right. \\
=\left(m^{2}+P\right)_{+}^{-r+\frac{1}{2}} \ln \left(m^{2}\right. & +P)_{+}-(-1)^{r} \pi\left(m^{2}+P\right)_{-}^{-r+\frac{1}{2}} \\
& \quad+(-1)^{r} i\left(m^{2}+P\right)_{-}^{-r+\frac{1}{2}} \ln \left(m^{2}+P\right)_{-}
\end{aligned}
$$

and

$$
\begin{array}{r}
\left(m^{2}+P+i 0\right)^{-r+\frac{1}{2}} \ln \left(m^{2}+P+i 0\right)=\left(m^{2}+P\right)_{+}^{-r+\frac{1}{2}} \ln \left(m^{2}+P\right)_{+} \\
-(-1)^{r} \pi\left(m^{2}+P\right)_{-}^{-r+\frac{1}{2}}-2(-1)^{r} \pi\left(m^{2}+P\right)_{-}^{-r+\frac{1}{2}} \ln \left(m^{2}+P\right)_{-} \\
+(-1)^{r} i\left(m^{2}+P\right)_{-}^{-r+\frac{1}{2}} \ln ^{2}\left(m^{2}+P\right) .
\end{array}
$$

By considering the formula ([5],p. 577, form (4.9))

$$
\left(m^{2}+P+i 0\right)^{-r}=\left(m^{2}+P\right)^{-r}-\frac{(-1)^{r-1} i \pi}{(r-1) !} \delta^{(r-1)}\left(m^{2}+P\right)
$$

we have

$$
\begin{aligned}
\left(m^{2}+P+i 0\right)^{-r} & \ln \left(m^{2}+P+i 0\right)=(-1)^{r} i \pi\left(m^{2}+P\right)_{-}^{-r} \\
& -\frac{(-1)^{r} \pi^{2} \delta^{(r-1)}\left(m^{2}+P\right)}{2(r-1) !}+\left(m^{2}+P\right)^{-r} \ln \left|m^{2}+P\right|
\end{aligned}
$$

and

$$
\begin{gathered}
\left(m^{2}+P+i 0\right)^{-r} \ln \left(m^{2}+P+i 0\right)=\frac{(-1)^{-r-1} i \pi^{3} \delta^{(r-1)}\left(m^{2}+P\right)}{3(r-1) !} \\
-(-1)^{r} \pi^{2}\left(m^{2}+P\right)_{-}^{-r}+2 i \pi(-1)^{r}\left(m^{2}+P\right)_{-}^{-r} \ln \left(m^{2}+P\right)_{-} \\
+\left(m^{2}+P\right)^{-r} \ln ^{2}\left|m^{2}+P\right|
\end{gathered}
$$




\section{Main Results}

Lemma 1. For $r$, and $s=1,2,3, \ldots$ we have

$$
\begin{gathered}
\left(m^{2}+P\right)^{-r}\left[\left(m^{2}+P\right)^{-s} \ln \left|m^{2}+P\right|\right]-\frac{(-1)^{s} \pi^{2}}{2(s-1) !}\left(m^{2}+P\right)^{-r} \delta^{(s-1)}\left(m^{2}+P\right)- \\
-\frac{(-1)^{r+s} \pi^{2}}{(r-1) !}\left(m^{2}+P\right)_{-}^{-s} \delta^{(r-1)}\left(m^{2}+P\right)= \\
\left(m^{2}+P\right)^{-r-s} \ln \left|m^{2}+P\right|-\frac{(-1)^{r+s} \pi^{2} \delta^{(r+s-1)}}{2(r+s-1) !}\left(m^{2}+P\right)
\end{gathered}
$$

and

$$
\begin{gathered}
\left(m^{2}+P\right)^{-r}\left[\left(m^{2}+P\right)^{-s} \ln \left|m^{2}+P\right|\right]+(-1)^{s} \pi^{2}\left(m^{2}+P\right)^{-r} \delta^{(s-1)}\left(m^{2}+P\right)+ \\
+\frac{(-1)^{r} \pi^{2}}{(r-1) !}\left(m^{2}+P\right)_{+}^{-s} \delta^{(r-1)}\left(m^{2}+P\right)= \\
\left(m^{2}+P\right)^{-r-s} \ln \left|m^{2}+P\right|-\frac{(-1)^{r+s} \pi^{2} \delta^{(r+s-1)}}{2(r+s+1) !}\left(m^{2}+P\right)
\end{gathered}
$$

Proof. From (1.12) we have

$$
\begin{gathered}
\left(m^{2}+P+i 0\right)^{-r}\left\{\left(m^{2}+P+i 0\right)^{-s} \ln \left(m^{2}+P+i 0\right)\right\}= \\
\left(m^{2}+P+i 0\right)^{-r-s} \ln \left(m^{2}+P+i 0\right)
\end{gathered}
$$

valid for $r, s=1,2, \ldots$

Taking into account the following formulas

$$
\left(m^{2}+P+i 0\right)^{-r}=\left(m^{2}+P\right)^{-r}-\frac{(-1)^{r-1} i \pi}{(r-1) !} \delta^{(r-1)}\left(m^{2}+P\right),
$$

$r=1,2, \ldots$ and

$$
\begin{gathered}
\left(m^{2}+P\right)^{-r} \ln \left(m^{+} P+i 0\right)=(-1)^{r} i \pi\left(m^{2}+P\right)_{-}^{-r} \\
-\frac{(-1)^{r} \pi \delta^{(r-1)}\left(m^{2}+P\right)}{2(r-1) !}+\left(m^{2}+P\right)^{-r} \ln \left|m^{2}+P\right|,
\end{gathered}
$$

$r=1,2, \ldots$, we can write $(2.3)$ in the equivalently form

$$
\left\{\left(m^{2}+P\right)^{-r}+\frac{(-1)^{r}}{(r-1) !} i \pi \delta^{(r-1)}\left(m^{2}+P\right)\right\} .
$$




$$
\begin{aligned}
& \left\{(-1)^{s} i \pi\left(m^{2}+P\right)_{-}^{-s}-\frac{(-1)^{s} \pi^{2}}{2(s-1) !} \delta^{(s-1)}\left(m^{2}+P\right)+\left(m^{2}+P\right)^{-s} \ln \left|m^{2}+P\right|\right\}= \\
& (-1)^{r+s} i \pi\left(m^{2}+P\right)_{-}^{-r-s}-\frac{(-1)^{r+s} \pi^{2}}{2(r+s+1) !} \delta^{(r+s+1)}\left(m^{2}+P\right)+\left(m^{2}+P\right)^{-r-s} \ln \left|m^{2}+P\right|
\end{aligned}
$$

valid for $r, s=1,2, \ldots$.

Expanding and equating the real parts of (2.6) we have

$$
\begin{gathered}
\left(m^{2}+P\right)^{-r}\left[\left(m^{2}+P\right)^{-s} \ln \left|m^{2}+P\right|\right]-\frac{(-1)^{s} \pi^{2}}{2(s-1) !}\left(m^{2}+P\right)^{-r} \delta^{(s-1)}\left(m^{2}+P\right)- \\
-\frac{(-1)^{r+s} \pi^{2}}{(r-1) !}\left(m^{2}+P\right)_{-}^{-s} \delta^{(r-1)}\left(m^{2}+P\right)= \\
\left(m^{2}+P\right)^{-r-s} \ln \left|m^{2}+P\right|-\frac{(-1)^{r+s} \pi^{2} \delta^{(r+s+1)}}{2(r+s-1) !}\left(m^{2}+P\right) .
\end{gathered}
$$

Replacing $\left(m^{2}+P\right)$ by $-\left(m^{2}+P\right)$ in the last equation we have

$$
\begin{gathered}
\left(m^{2}+P\right)^{-r}\left[\left(m^{2}+P\right)^{-s} \ln \left|m^{2}+P\right|\right]+\frac{(-1)^{s} \pi^{2}}{2(s-1) !}\left(m^{2}+P\right)^{-r} \delta^{(s-1)}\left(m^{2}+P\right)+ \\
+\frac{(-1)^{r+s} \pi^{2}}{(r-1) !}\left(m^{2}+P\right)_{+}^{-s} \delta^{(r-1)}\left(m^{2}+P\right)= \\
\left(m^{2}+P\right)^{-r-s} \ln \left|m^{2}+P\right|-\frac{(-1)^{r+s} \pi^{2} \delta^{(r+s+1)}}{2(r+s-1) !}\left(m^{2}+P\right) .
\end{gathered}
$$

Lemma 2. For $r, s=1,2, \ldots$, we have

$$
\begin{gathered}
2\left(m^{2}+P\right)^{-r}\left[\left(m^{2}+P\right)^{-s} \ln \left|m^{2}+P\right|\right]+\frac{(-1)^{r} \pi^{2}}{(r-1) !} . \\
{\left[\operatorname{sgn}\left(m^{2}+P\right)\left(m^{2}+P\right)^{-s}\right] \delta(r-1)\left(m^{2}+P\right)=} \\
2\left(m^{2}+P\right)^{-r-s} \ln \left|m^{2}+P\right|
\end{gathered}
$$

and

$$
\begin{gathered}
(-1)^{s}\left(m^{2}+P\right)^{-r}\left(m^{2}+P\right)_{-}^{-s}-\frac{(-1)^{r+s} \pi^{2}}{2(r-1) !(s-1) !} \delta^{(r-1)}\left(m^{2}+P\right) . \\
\delta^{(s-1)}\left(m^{2}+P\right)+\frac{(-1)^{r}}{(r-1) !}\left[\left(m^{2}+P\right)^{-s} \ln \left|m^{2}+P\right|\right] \delta^{(r-1)}\left(m^{2}+P\right)= \\
(-1)^{r+s}\left(m^{2}+P\right)_{-}^{-r+s} .
\end{gathered}
$$


Proof. Adding the equations (2.1) and (2.2) we have

$$
\begin{gathered}
2\left(m^{2}+P\right)^{-r}\left[\left(m^{2}+P\right)^{-s} \ln \left|m^{2}+P\right|\right]+\frac{(-1)^{r} \pi^{2}}{(r-1) !} \\
{\left[\operatorname{sgn}\left(m^{2}+P\right)\left(m^{2}+P\right)^{-s}\right] \delta(r-1)\left(m^{2}+P\right)=} \\
2\left(m^{2}+P\right)^{-r-s} \ln \left|m^{2}+P\right|
\end{gathered}
$$

Expanding and equating the imaginary parts of the equation (2.6) we obtain

$$
\begin{gathered}
(-1)^{s}\left(m^{2}+P\right)^{-r}\left(m^{2}+P\right)_{-}^{-s}-\frac{(-1)^{r+s} \pi^{2}}{2(r-1) !(s-1) !} \delta^{(r-1)}\left(m^{2}+P\right) . \\
\delta^{(s-1)}\left(m^{2}+P\right)+\frac{(-1)^{r}}{(r-1) !}\left[\left(m^{2}+P\right)^{-s} \ln \left|m^{2}+P\right|\right] \delta^{(r-1)}\left(m^{2}+P\right)= \\
(-1)^{r+s}\left(m^{2}+P\right)_{-}^{-r+s} .
\end{gathered}
$$

Lemma 3. For $r, s=1,2, \ldots$ it result

$$
\begin{aligned}
& \left(m^{2}+P\right)^{-r}\left(m^{2}+P\right)_{+}^{-s}-\frac{(-1)^{r+s} \pi^{2}}{2(r-1) !(s-1) !} \delta^{(r-1)}\left(m^{2}+P\right) . \\
& \left.\delta^{(} r-1\right)\left(m^{2}+P\right)+\frac{(-1)^{r}}{(r-1) !}\left[\left(m^{+} P\right)^{-s} \ln \left|m^{2}+P\right|\right] \delta^{(r-1)}\left(m^{2}+P\right)= \\
& \left(m^{2}+P\right)_{+}^{-r-s}
\end{aligned}
$$

and

$$
\begin{gathered}
\left(m^{2}+P\right)^{-r}\left[\operatorname{sgn}\left(m^{2}+P\right)\left(m^{2}+P\right)^{-s}\right]-\frac{2(-1)^{r}}{(r-1) !} . \\
{\left[\left(m^{+} P\right)^{-s} \ln \left|m^{2}+P\right|\right] \delta^{(r-1)}\left(m^{2}+P\right)=} \\
\operatorname{sgn}\left(m^{2}+P\right)\left(m^{2}+P\right)^{-r-s}
\end{gathered}
$$

Proof. Replacing $\left(m^{2}+P\right)$ by $-\left(m^{2}+P\right)$ in the equation (2.8) we have (2.9); and subtracting (2.9) from (2.8) we have (2.10).

Lemma 4. For $r, s=1,2, \ldots$, we have

$$
\begin{gathered}
\left(m^{2}+P\right)^{-r}\left[\left(m^{2}+P\right)^{s} \ln ^{2}\left|m^{+} P\right|\right]-(-1)^{s} \pi^{2}\left(m^{2}+P\right)^{-r}\left(m^{2}+P\right)_{-}^{s}- \\
-\frac{2(-1)^{r+s}}{(r-1) !} \pi^{2}\left[\left(m^{2}+P\right)_{-}^{s} \ln \left(m^{2}+P\right)_{-}\right] \delta^{(r-1)}\left(m^{2}+P\right)=
\end{gathered}
$$




$$
\left(m^{2}+P\right)^{-r+s} \ln ^{2}\left|m^{2}+P\right|-(-1)^{r+s} \pi^{2}\left(m^{2}+P\right)_{-}^{-r+s}
$$

and

$$
\begin{gathered}
\left(m^{2}+P\right)^{-r}\left[\left(m^{2}+P\right)^{s} \ln ^{2}\left|m^{+} P\right|\right]-\pi^{2}\left(m^{2}+P\right)^{-r}\left(m^{2}+P\right)_{+}^{s}+ \\
+\frac{2(-1)^{r}}{(r-1) !} \pi^{2}\left[\left(m^{2}+P\right)_{+}^{s} \ln \left(m^{2}+P\right)_{+}\right] \delta^{(r-1)}\left(m^{2}+P\right)= \\
\left(m^{2}+P\right)^{-r+s} \ln ^{2}\left|m^{2}+P\right|-\pi^{2}\left(m^{2}+P\right)_{+}^{-r+s}
\end{gathered}
$$

Proof. Taking into account the equation (1.12) we have

$$
\begin{gathered}
\left(m^{2}+P+i 0\right)^{-r}\left\{\left(m^{2}+P+i 0\right)^{s} \ln ^{2}\left(m^{2}+P+i 0\right)\right\}= \\
\left(m^{2}+P+i 0\right)^{-r+s} \ln ^{2}\left(m^{2}+P+i 0\right)
\end{gathered}
$$

valid for $r, s=1,2, \ldots$

Equivalently, from (1.17) and (1.22) we can write

$$
\begin{gathered}
\left\{\left(m^{2}+P\right)^{-r}+\frac{(-1)^{r} i \pi}{(r-1) !} \delta^{(r-1)}\left(m^{2}+P\right)\right\} \\
\left\{\left(m^{2}+P\right)^{s} \ln ^{2}\left|m^{2}+P\right|-(-1)^{s} \pi^{2}\left(m^{2}+P\right)_{-}^{s}+2(-1)^{s} i \pi\left(m^{2}+P\right)_{-}^{s} \ln \left(m^{2}+P\right)_{-}\right\}= \\
\left({ }^{2}+P\right)^{-r+s} \ln ^{2}\left|m^{2}+P\right|-(-1)^{r+s} \pi^{2}\left(m^{2}+P\right)_{-}^{r+s}+ \\
2(-1)^{r+s} i \pi\left(m^{2}+P\right)_{-}^{-r+s} \ln \left(m^{2}+P\right)_{-} \\
-\frac{(-1)^{r+s} i \pi^{3}}{3(r-s-1) !} \delta^{(r-s-1)}\left(m^{2}+P\right)
\end{gathered}
$$

valid for $r, s=1,2, \ldots$

Expanding the equation (2.14) and equating the real parts, it results (2.11), and replacing $\left(m^{2}+P\right)$ for $-\left(m^{2}+P\right)$ we have $(2.12)$.

Lemma 5. For $r, s=1,2, \ldots$, :

$$
\begin{aligned}
&\left(m^{2}+P\right)^{-r} {\left[\operatorname{sgn}\left(m^{2}+P\right)\left(m^{2}+P\right)^{s}\right] } \\
&-\frac{2(-1)^{r}}{(r-1) !}\left[\left(m^{2}+P\right)^{s} \ln \left|m^{2}+P\right| \delta^{(r-1)}\left(m^{2}+P\right)\right] \\
&=\operatorname{sgn}\left(m^{2}+P\right)\left(m^{2}+P\right)^{-r+s}
\end{aligned}
$$

and

$$
\left(m^{2}+P\right)^{-r}\left[\left(m^{2}+P\right)^{r} \ln ^{2}\left|m^{2}+P\right|\right]
$$




$$
\begin{aligned}
+\frac{(-1)^{r} \pi^{2}}{(r-1) !}\left[\operatorname{sgn}\left(m^{2}+P\right) \ln \left|m^{2}+P\right|\right] \delta^{(r-1)}\left(m^{2}+P\right) \\
=\left(m^{2}+P\right)^{-r+s} \ln ^{2}\left|m^{2}+P\right|
\end{aligned}
$$

Proof. Subtracting (2.12) from (2.11) we obtain (2.15). Adding the equation (2.12) to (2.11) results $(2.16)$.

Lemma 6. For $r, s=1,2, \ldots$, it results

$$
\begin{gathered}
2(-1)^{s}\left(m^{2}+P\right)^{-r}\left[\left(m^{2}+P\right)_{-}^{s} \ln \left(m^{2}+P\right)_{-}\right]+ \\
\frac{(-1)^{r}}{(r-1) !}\left[\left(m^{2}+P\right)^{s} \ln ^{2}\left|m^{2}+P\right|\right] \cdot \delta^{(r-1)}\left(m^{2}+P\right)- \\
-\frac{(-1)^{r+s} \pi^{2}}{(r-1) !}\left(m^{2}+P\right)_{-}^{s} \delta^{(r-1)}\left(m^{2}+P\right)= \\
2(-1)^{r+s}\left(m^{2}+P\right)_{-}^{-r+s} \ln \left(m^{2}+P\right)_{-} \frac{(-1)^{r+s} \pi^{2}}{3(r-s-1) !} \delta^{(r-s-1)}\left(m^{2}+P\right)
\end{gathered}
$$

and

$$
\begin{gathered}
2\left(m^{2}+P\right)^{-r}\left[\left(m^{2}+P\right)_{+}^{s} \ln \left(m^{2}+P\right)_{+}\right] \\
-\frac{(-1)^{r}}{(r-1) !}\left[\left(m^{2}+P\right)^{s} \ln ^{2}\left|m^{2}+P\right|\right] . \delta^{(r-1)}\left(m^{2}+P\right) \\
+\frac{(-1)^{r} \pi^{2}}{(r-1) !}\left(m^{2}+P\right)_{+}^{s}\left(m^{2}+P\right) \\
=2\left(m^{2}+P\right)_{+}^{-r+s} \ln \left(m^{2}+P\right)_{+}+\frac{(-1)^{r+s} \pi^{2}}{3(r-s-1) !} \delta^{(r-s-1)}\left(m^{2}+P\right) .
\end{gathered}
$$

Proof. Equating the imaginary parts of (2.14) we have (2.17), and replacing $\left(m^{2}+P\right)$ for $-\left(m^{2}+P\right)$ in (2.17) we obtain (2.18).

Lemma 7. For $r, s=1,2, \ldots$, we have

$$
\begin{aligned}
& 2\left(m^{2}+P\right)^{-r} {\left[\left(m^{2}+P\right)^{s} \ln \left|m^{2}+P\right|\right] } \\
&+\frac{(-1)^{r} \pi^{2}}{(r-1) !}\left[\operatorname{sgn}\left(m^{2}+P\right)\left(m^{2}+P\right)^{s}\right] \delta^{(r-1)}\left(m^{2}+P\right) \\
&=2\left(m^{2}+P\right)^{-r+s} \ln \left|m^{2}+P\right|
\end{aligned}
$$

and 


$$
\begin{aligned}
\left(m^{2}+P\right)^{-r}\left[\operatorname{sgn}\left(m^{2}+P\right)\left(m^{2}+P\right)^{s} \ln \left|m^{2}+P\right|\right] & \\
-\frac{(-1)^{r}}{(r-1) !} & {\left[\left(m^{2}+P\right)^{s} \ln ^{2}\left|m^{2}+P\right|\right] \delta^{(r-1)}\left(m^{2}+P\right) } \\
= & \frac{(-1)^{r+s} \pi^{2}}{6(r-s-1) !} \delta^{(r-s-1)}\left(m^{2}+P\right) \\
\quad & \quad \operatorname{sgn}\left(m^{2}+P\right)\left(m^{2}+P\right)^{-r+s} \ln \left|m^{2}+P\right| .
\end{aligned}
$$

Proof. Adding the equation (2.17) to the equation (2.18) result (2.19) and subtracting (2.17) to the equation (2.18) we obtain (2.20).

\section{References}

[1] A. Gonzalez Domínguez, On some heterodox distributional multiplicative products, Trabajos de Matemática, Instituto Argentino de MatemáticaCONICET, 17 (1978).

[2] B. Fischer, Some result on convergent and divergent products of distributions, Bull. Math. de la Soc. Sci. Math. de la R. S. de Roumanie, 22, No. 1 (1978), 3-13.

[3] C.K. Li, A Review on the Productos of Distributions, Mathematical Methods in Engineering, Springer (2007).

[4] I.M. Gelfand, G.E. Shilov, Generalized Functions, Volume I, Academic Press, New York (1964).

[5] D.W. Bresters, On distributions connected with quadratic form, SIAM J. Appl. Math., 16 (1968), 563-581, doi: 10.1137/0116045.

[6] S.E. Trione, Distributional Products. Cursos de Matemática, No. 3. Instituto Argentino de Matemática-CONICET. Argentina (1980).

[7] S.E. Trione, Distributional Multiplicative Products, Trabajos de Matemática, No. 22, Instituto Argentino de Matemática-CONICET (1979). 
